University of Wollongong

Research Online

Faculty of Engineering and Information

Faculty of Engineering and Information

Sciences - Papers: Part A

Sciences

$1-1-2015$

\title{
A tensor-based subspace wall clutter mitigation method for through-the- wall radar imaging
}

Fok Hing Chi Tivive

University of Wollongong, tivive@uow.edu.au

Abdesselam Bouzerdoum

University of Wollongong, bouzer@uow.edu.au

Follow this and additional works at: https://ro.uow.edu.au/eispapers

Part of the Engineering Commons, and the Science and Technology Studies Commons

Research Online is the open access institutional repository for the University of Wollongong. For further information contact the UOW Library: research-pubs@uow.edu.au 


\title{
A tensor-based subspace wall clutter mitigation method for through-the-wall radar imaging
}

\author{
Abstract \\ In through-the-wall radar imaging, targets behind the wall reflect weak electromagnetic signals that are \\ obscured by the strong returns from exterior wall, rendering the detection and classification of indoor \\ stationary targets very difficult. In this paper, a tensor-based subspace method is proposed for wall clutter \\ mitigation. The radar signals received from the antenna array are transformed into a data tensor. Higher- \\ order singular value decomposition is used to segregate the wall reflections from the target returns. \\ Simulation and experimental results show that the proposed method is effective in removing reflections \\ backscattered from both homogeneous and heterogeneous walls.

\section{Keywords} \\ wall, imaging, radar, subspace, method, tensor, mitigation, clutter

\section{Disciplines} \\ Engineering | Science and Technology Studies

\section{Publication Details} \\ F. Hing Chi. Tivive \& A. Bouzerdoum, "A tensor-based subspace wall clutter mitigation method for through- \\ the-wall radar imaging," in in Acoustics, Speech and Signal Processing (ICASSP), 2015 IEEE International \\ Conference on, 2015, pp. 2449-2453.
}




\title{
A TENSOR-BASED SUBSPACE WALL CLUTTER MITIGATION METHOD FOR THROUGH-THE-WALL RADAR IMAGING
}

\author{
Fok Hing Chi Tivive and Abdesselam Bouzerdoum \\ School of Electrical, Computer and Telecommunications Engineering, \\ University of Wollongong, \\ Northfields Avenue, Wollongong NSW 2522, Australia \\ (e-mail: $\{$ tivive, bouzer $\} @$ uow.edu.au)
}

\begin{abstract}
In through-the-wall radar imaging, targets behind the wall reflect weak electromagnetic signals that are obscured by the strong returns from exterior wall, rendering the detection and classification of indoor stationary targets very difficult. In this paper, a tensor-based subspace method is proposed for wall clutter mitigation. The radar signals received from the antenna array are transformed into a data tensor. Higher-order singular value decomposition is used to segregate the wall reflections from the target returns. Simulation and experimental results show that the proposed method is effective in removing reflections backscattered from both homogeneous and heterogeneous walls.
\end{abstract}

Index Terms- Wall clutter mitigation, Tensor, HOSVD, Subspace projection, Through-the-Wall radar imaging.

\section{INTRODUCTION}

With recent advances in radar technology, imaging through opaque obstacles, such as walls and doors, has attracted interest from researchers, research organizations, government agencies, and the military. The objectives of through-the-wall radar imaging (TWRI) are to detect, locate, and track targets inside an enclosed building structure and determine the building layouts [1-7]. One of the main problems facing TWRI is the strong and persistent reflections backscattered from the exterior wall, which mask the weak target returns, resulting in images with high clutter. For imaging of moving targets behind walls, change detection or Doppler processing can be applied to remove the wall contributions [8-10]. However, for stationary targets, mitigation of the wall clutter is much more challenging.

Several wall clutter mitigation methods have been proposed for TWRI [11-22]. A simple method for wall removal is background subtraction; however, it requires prior measurements from the background or reference scene devoid of target(s) to eliminate the overwhelming electromagnetic (EM) wall signature. Though this method is effective, accessibility to the background scene is not always possible in practice. Another method for wall clutter mitigation is to estimate the wall parameters so as to model the wall returns, which are then subtracted from the total radar returns to obtain a wall clutter free signal $[11,12]$. This method works well if the wall parameter estimation and modeling are done correctly. In [13], the wall reflections are removed by employing three parallel antenna arrays, where the upper and lower ones act as receiver and the middle one as transmitter. The wall contributions is removed by subtracting the radar returns from the lower and upper arrays. This method, however, requires an additional array of receivers, and it is difficult to control the effect of the subtraction operation on the target reflections. Recently, several methods have been proposed for wall clutter mitigation, which do not depend on the wall parameter estimation nor require prior knowledge of the background scene [14-22]. In [14], spatial filtering was proposed to cancel the wall reflections by removing the DC (direct-current) value or low spatial frequencies across the antenna array [14]. This method works well when the wall returns are the same at all antenna locations and the wall is homogeneous. In [15-20], singular value decomposition (SVD) was used to segregate the wall reflections from the target returns. In $[15,16]$, the SVD approach was applied to range profiles, in [17, 19,20] SVD was applied to the data matrix comprising the space-frequency measurements, and in [18] SVD was applied to the formed image. More recently, a wall clutter removal method based on entropy was proposed in [21]. It assumes that the antenna array is placed parallel to the wall surface and the wall clutter has similar behavior over each signal trace. In [22], the mitigation of wall returns from the radar signal was solved as a sparse representation problem, where the discrete prolate spheroidal sequence (DPSS) is used to estimate the wall returns from compressive measurements. This method depends on a predefined range, obtained from EM simulations [23], to determine the recovered wall coefficients.

Many of the existing wall clutter mitigation methods assume that the wall is homogeneous and the antenna array is aligned perfectly parallel to the wall surface. However, these two conditions may not always be satisfied in practical TWRI applications. Furthermore, the study conducted in $[17,20]$ shows that the wall returns can span a multidimensional subspace, due to, among other factors, the wall EM characteristics, the wall thickness uniformity, and the configuration of the antenna array. In this paper, we propose to use higherorder SVD (HOSVD) [24] for wall clutter mitigation. Since its introduction in the mid-1990's, HOSVD has been applied to various research areas, including parameter estimation [25], image denoising [26], text representation [27], and web link analysis [28]. Here, it is used to develop a method that can effectively remove the wall returns from the received signals. The proposed method assumes that the reflections backscattered from the front wall are relatively much stronger than those backscattered from the behind-the-wall targets. Furthermore, the wall and target reflections reside in different subspaces spanned by singular vectors extracted from the data tensor.

The remainder of the paper is organized as follows. Section 2 presents TWRI signal model and image formation using delay-andsum (DS) beamforming. Section 3 describes the proposed wall clutter mitigation method using HOSVD. Section 4 discusses the experimental results and comparisons based on simulated and real data. Section 5 presents concluding remarks. 


\section{SIGNAL MODEL AND DS BEAMFORMING FOR TWRI}

Consider an $N$-element linear synthetic aperture radar (SAR) for TWRI. The transceiver emits a wideband stepped-frequency signal comprising $M$ frequencies, $\omega_{m}(m=1, \ldots, M)$. The radar is placed at a certain standoff distance from a wall. Suppose there are $G$ targets behind the wall, the radar signal $y_{n}(m)$ of the $m$ th frequency received by the $n$th antenna can be expressed as

$$
y_{n}(m)=\sum_{r=1}^{R} \sigma_{\mathrm{w}} A_{r} \exp \left(-j \omega_{m} \tau_{\mathrm{w}, n}^{r}\right)+\sum_{p=1}^{G} \sigma_{p} \exp \left(-j \omega_{m} \tau_{p, n}\right),
$$

where $\sigma_{\mathrm{w}, n}$ is the complex reflectivity of the wall at the $n$th antenna location, $R$ is the number of wall reverberations, $\tau_{\mathrm{w}, n}^{1}$ is the propagation delay associated with the direct return from the wall, $\tau_{\mathrm{w}, n}^{r}$, $r>1$ is the propagation delay associated with the $r$ th wall reverberation, $A_{r}$ is the path loss factor associated with the $r$ th wall return, $\sigma_{p}$ is the complex reflectivity of the $p$ th target, and $\tau_{p, n}$ is the twoway propagation delay between the $n$th antenna and the $p$ th target.

To form an image, the scene is divided into a rectangular grid consisting of $Q$ pixels. Using DS beamforming, the complex amplitude of the $q$ th pixel can be computed as

$$
I(q)=\frac{1}{N M} \sum_{n=1}^{N} \sum_{m=1}^{M} y_{n}(m) \exp \left(j \omega_{m} \tau_{n, q}\right)
$$

where $\tau_{n, q}$ denotes the focusing delay between the $n$th antenna and the $q$ th pixel; see [29] for more details on computation the focussing delay. Applying DS beamforming directly to the radar signal given in (1) produces images with high wall clutter. Therefore, wall clutter mitigation is required to remove the wall returns from the received signals. The next section describes the proposed tensor-based subspace method for wall clutter mitigation.

\section{WALL CLUTTER MITIGATION USING HOSVD}

In [17] and [20], we have shown that the direct wall returns and the wall reverberations reside in a multidimensional subspace and used SVD to identify the singular vectors spanning the wall subspace. However, not all wall returns are captured by the dominant singular vectors. Some wall residual reflections interact with the target and form singular vectors which carry information about the target and the wall. To address this problem, we developed a segmentation technique based on Gaussian Mixture model (GMM) to remove the weak wall components from the target signals [19]. Here, we employ HOSVD to determine a multilinear subspace characterizing the wall returns and then project the radar signals onto the subspace orthogonal to the multilinear wall subspace. After wall clutter mitigation, we use DS beamforming to form the image of the scene.

\subsection{Multilinear Wall Subspace}

The multilinear wall subspace is determined by applying HOSVD to the data tensor, which is obtained by arranging the frequency samples from each antenna into a Hankel matrix $Y_{n}$ [24]:

$$
Y_{n}=\left(\begin{array}{cccc}
y_{n}(1) & y_{n}(2) & \ldots & y_{n}(L) \\
y_{n}(2) & y_{n}(3) & \ldots & y_{n}(L+1) \\
\vdots & \vdots & \ddots & \vdots \\
y_{n}(L) & y_{n}(L+1) & \ldots & y_{n}(2 L-1)
\end{array}\right)
$$

where $L=\lceil M / 2\rceil$. The $N$ Hankel matrices are then stacked one behind the other to form a mode-3 tensor, $\mathcal{Y}=\left\{Y_{1}, \ldots, Y_{N}\right\} \in$ $\mathbb{C}^{L \times L \times N}$. Using HOSVD, the tensor $\mathcal{Y}$ can be decomposed as

$$
\mathcal{Y}=\mathcal{C} \times{ }_{1} U^{(1)} \times{ }_{2} U^{(2)} \times{ }_{3} U^{(3)},
$$

where $\mathcal{C}$ is the core tensor, $\times_{i}, i=1,2,3$ is the mode- $i$ product, $U^{(i)}=\left[\boldsymbol{u}_{1}^{(i)}, \ldots, \boldsymbol{u}_{k_{i}}^{(i)}\right]$ is the unitary matrix of mode- $i$ matricization of tensor $\mathcal{Y}$, and $k_{i}$ is the number of singular vectors associated with mode- $i$. Though it is expected that the strong wall reflections span the first few dominant singular vectors, some weak wall components may reside in the non-dominant singular vectors, which are interleaved with the target singular vectors $[19,20]$. Therefore, the identification of the singular vectors spanning the multilinear wall subspace is performed as follows. First, a vector space $S^{i, j}$ is formed with the $j$ th singular vector of mode- $i$ :

$$
S^{i, j}=\boldsymbol{u}_{j}^{(i)}\left(\boldsymbol{u}_{j}^{(i)}\right)^{H} .
$$

Next, the data tensor $\mathcal{Y}$ is projected onto the vector space $S^{i, j}$

$$
\widehat{\mathcal{Y}}^{i, j}=\mathcal{Y} \times_{i} S^{i, j}
$$

Let $\widehat{Y}_{n}^{i, j}$ denote the processed Hankel matrix associated with the $n$th antenna obtained from the data tensor $\widehat{\mathcal{Y}}^{i, j}=\left\{\widehat{Y}_{1}^{i, j}, \ldots, \widehat{Y}_{N}^{i, j}\right\}$ and $z_{k l}$ denote the element at the $k$ th row and $l$ th column of matrix $\widehat{Y}_{n}^{i, j}$. The radar signal $\widehat{y}_{n}^{i, j}(m)$ of the $m$ th frequency received at the $n$th antenna location is obtained by performing a diagonal averaging operation given as follows:

$\widehat{y}_{n}^{i, j}(m)= \begin{cases}\frac{1}{m} \sum_{l=1}^{m} z_{l, m-l+1}, & \text { for } 1 \leq m \leq L \\ \frac{1}{N-L+1} \sum_{l=m-L+1}^{N-L+1} z_{l, m-l+1}, & \text { for } L+1 \leq m \leq M\end{cases}$

The singular vectors spanning the multilinear subspace can be identified from the range profiles obtained from the reconstructed signals. In [20], we developed a technique to classify the singular vector into wall and target classes. Here, this classification technique is applied to each mode to determine the multilinear wall subspace. The next step is to project the data tensor onto the subspace orthogonal to the wall subspace for wall clutter mitigation.

\subsection{Wall Clutter Mitigation by Subspace Projection}

First the multilinear wall subspace is formed, and then a subspace projection is performed for wall clutter removal. Let $W^{i}$ be the index set of wall singular vector associated with mode- $i$ and $\widehat{U}^{(i)}=$ $\left\{\mathbf{u}_{j}^{(i)}\right\}_{j \in W^{i}}$ be a matrix of singular vectors formed from the index set of $W^{i}$. The subspace orthogonal to the wall subspace of mode- $i$ is computed as

$$
P_{\perp}^{(i)}=I-\widehat{U}^{(i)}\left(\widehat{U}^{(i)}\right)^{H}
$$

where $I$ denotes the identity matrix. For wall clutter mitigation, the data tensor $\mathcal{Y}$ is projected onto the multilinear orthogonal subspace

$$
\widetilde{\mathcal{Y}}=\mathcal{Y} \times{ }_{1} P_{\perp}^{(1)} \times_{2} P_{\perp}^{(2)} \times_{3} P_{\perp}^{(3)} .
$$

The radar signals are reconstructed from the tensor $\widetilde{\mathcal{Y}}$ using Eq. (7), and then DS beamforming is applied to form the image of the scene. 


\section{RESULTS AND DISCUSSIONS}

Two sets of experiments are performed to validate the proposed wall clutter mitigation method using simulated and real data. For comparison purposes, five different wall clutter mitigation techniques are tested, namely background subtraction, spatial filtering, entropybased technique, SVD-based method, and DPSS-based method. The effectiveness of wall clutter mitigation is measured using the improvement factor (IF):

$$
\mathrm{IF}=10 \log \left(\frac{\mathrm{TCR}_{o}}{\mathrm{TCR}_{i}}\right)
$$

where $\mathrm{TCR}_{o}$ and $\mathrm{TCR}_{i}$ are, respectively, the target-to-clutter ratios of the formed image with and without wall clutter mitigation. The TCR of a radar image is calculated as

$$
\mathrm{TCR}=\frac{\frac{1}{N_{t}} \sum_{q \in A_{t}}|I(q)|^{2}}{\frac{1}{N_{c}} \sum_{q \in A_{c}}|I(q)|^{2}},
$$

where $A_{t}$ is the target region, $A_{c}$ is the clutter region defined as the entire image excluding the target region, and $N_{c}$ and $N_{t}$ are the number of pixels in the clutter and target regions, respectively.

\subsection{Simulated Scene}

The proposed method is tested using a simulated TWRI scene containing two targets behind a wall. The simulations are performed using XFDTD software. A 31-element antenna array of length $1.2 \mathrm{~m}$ is synthesized for TWRI. A homogeneous wall of thickness $1.5 \mathrm{~m}$ and a dielectric constant 7.76 is placed in front of the radar at a standoff distance of $1.5 \mathrm{~m}$. Since it is difficult to have perfect alignment between the antenna array and the wall surface in practice, the antenna array is deliberately tilted at an angle of $5^{\circ}$ with respect to the wall surface. Two square dihedrals of area $0.16 \mathrm{~m}^{2}$ are placed at (-0.6 1.6) and $(0.6,0.8) \mathrm{m}$ behind the wall. The scene behind the wall is interrogated using a modulated Gaussian pulse with a center frequency of $1.5 \mathrm{GHz}$ as an excitation signal. The time domain response is then converted into a stepped-frequency signal with 201 frequencies covering the frequency band [2,3] GHz.

Figure 1 depicts the images obtained after performing wall clutter mitigation using different methods. All six wall clutter mitigation methods suppress the strong wall returns, but some methods achieve clearer images than others. Background subtraction produces the least cluttered image, Fig. 1(a), whereas spatial filtering yields an image with the most wall clutter, Fig. 1(b). Spatial filtering performs poorly because the antenna array is not perfectly parallel to the wall surface. The entropy-based method produces an image with residual wall reverberations, due to misalignment of the antenna, Fig. 1(c). On the other hand, the DPSS-based method successfully remove the wall returns and the reflections from the closeby target, Fig. 1(d). This is because any reflections located between the antenna position and a distance of $1.5 \mathrm{~m}$ away from the front surface of the wall are removed. The subspace approaches based on SVD and HOSVD are very effective in removing the wall returns, Figs. 1(e) and (f), with the latter producing a much clearer image. The IFs of the formed images depicted in Fig. 1 are listed in the second column of Table 1. Clearly, background subtraction achieves the highest IF, followed by the proposed tensor-based method. Next, the wall clutter mitigation methods are tested using real radar measurements.

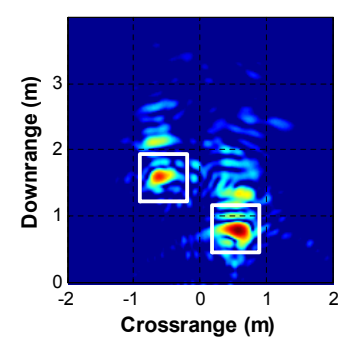

(a)

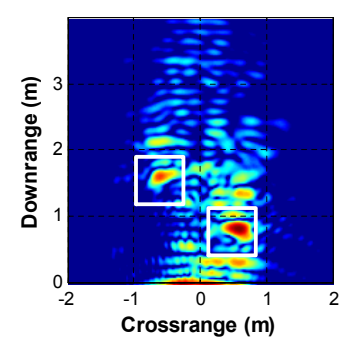

(c)

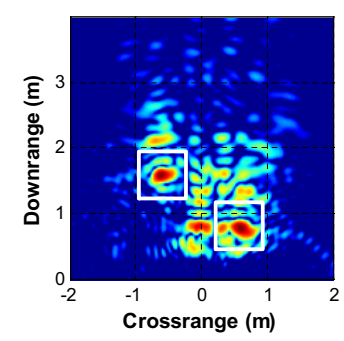

(e)

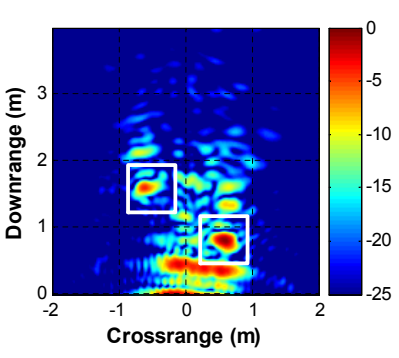

(b)

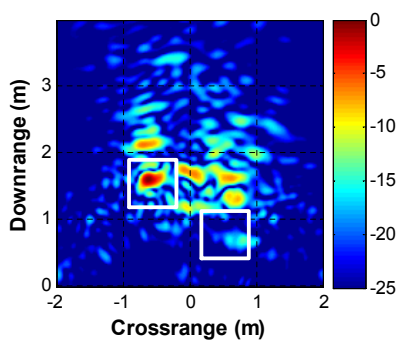

(d)

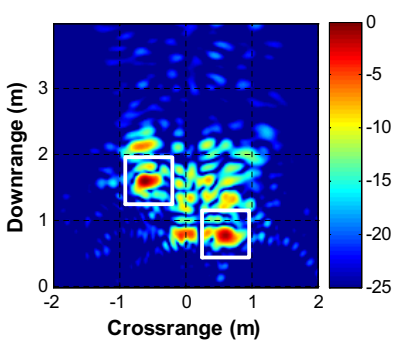

(f)
Fig. 1. Images of the synthesized scene obtained using different wall clutter mitigation methods: (a) background subtraction, (b) spatial filtering, (c) entropy-based method, (d) DPSS-based method, (e) SVD-based method and (f) proposed tensor-based method. The target is circled by a white rectangle.

Table 1. Improvement factor of the wall clutter mitigation methods.

\begin{tabular}{|l|c|c|}
\hline Approach & Simulated scene & Drywall scene \\
\hline Proposed method & $7.36 \mathrm{~dB}$ & $24.86 \mathrm{~dB}$ \\
\hline Background subtraction & $10.44 \mathrm{~dB}$ & $24.50 \mathrm{~dB}$ \\
\hline SVD-based method [19] & $7.01 \mathrm{~dB}$ & $25.34 \mathrm{~dB}$ \\
\hline Spatial filtering [14] & $3.96 \mathrm{~dB}$ & $10.24 \mathrm{~dB}$ \\
\hline Entropy time gating [21] & $5.67 \mathrm{~dB}$ & $4.66 \mathrm{~dB}$ \\
\hline DPSS-based method [22] & $4.44 \mathrm{~dB}$ & $25.46 \mathrm{~dB}$ \\
\hline
\end{tabular}

\subsection{Real TWRI Scene}

Radar signals are collected from a TWRI system in the Radar Imaging Lab of the Center for Advanced Communications at Villanova University. The TWRI scene comprises a drywall, which is built from a wooden frame, plywood, and gypsum wallboard. The scene comprises three dihedrals, four trihedrals, a sphere, and a tophat placed at different locations behind the wall, as shown in Fig. 2. A 69-antenna array of length $1.5 \mathrm{~m}$ is used to interrogate the scene with a stepped-frequency signal of $1 \mathrm{GHz}$ bandwidth centered at $2.5 \mathrm{GHz}$. A full description of the experimental setup and the specification of the imaging system is given in [30]. 


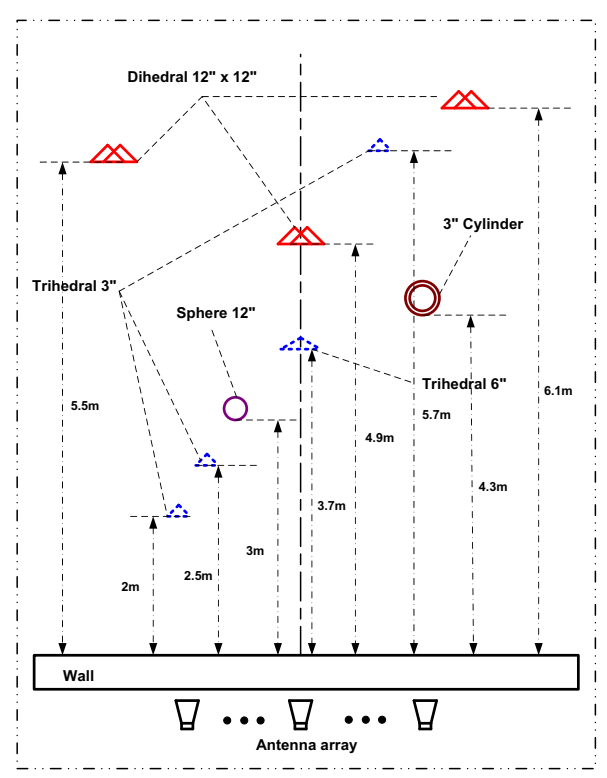

Fig. 2. Ground-truth image of the real TWRI scene.

The six wall clutter mitigation methods were tested on the real radar data. Figure. 3 presents the beamformed images obtained using different wall clutter mitigation methods. Spatial filtering and the entropy-based method fail to reveal all nine targets, Figs. 3(b) and (c). This is because the wall is heterogeneous, hence invalidating the assumption that the EM wall signature are invariant with antenna location. The other wall clutter mitigation methods are all effective in suppressing the wall returns. In the third column of Table 1, the DPSS- and SVD-based methods achieve slightly better IF than the proposed tensor-based method as both methods employ a threshold technique to remove the wall reflections. If the same threshold used in the DPSS-based technique is applied to HOSVD, the IF of the proposed tensor-based method improves to $26.55 \mathrm{~dB}$.

\section{CONCLUSION}

A tensor-based subspace projection method was proposed for TWRI to mitigate the wall clutter induced by both homogeneous and heterogenous walls. The radar signals received by the antenna array are converted into Hankel matrices, which are then stacked one behind the other to form a tensor. HOSVD and a classification technique are then employed to determine the singular vectors spanning the multilinear wall subspace. For wall clutter mitigation, the data tensor is projected onto the subspace orthogonal to the wall subspace. Experimental results using simulated and real data demonstrate that the proposed method can be very effective in removing wall clutter without relying on prior knowledge of the background scene.

\section{Acknowledgment}

The authors would like to thank Prof. Moeness Amin and Dr. Fauzia Ahmad from the Center of Advanced Communications at Villanova University, Villanova, PA, USA, for providing the experimental data. This work is supported by a grant from the Australian Research Council (ARC).

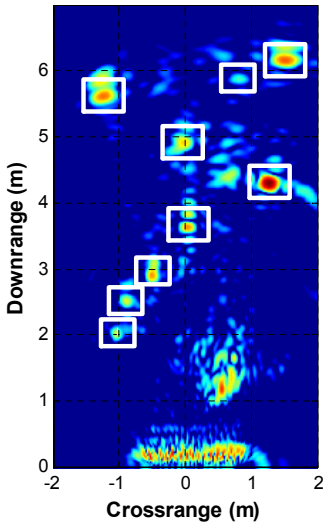

(a)

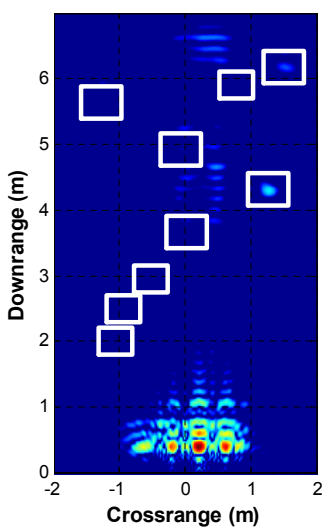

(c)

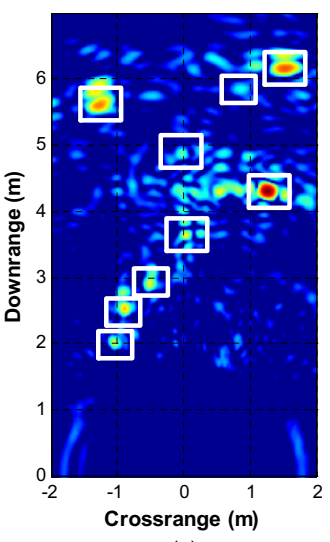

(e)

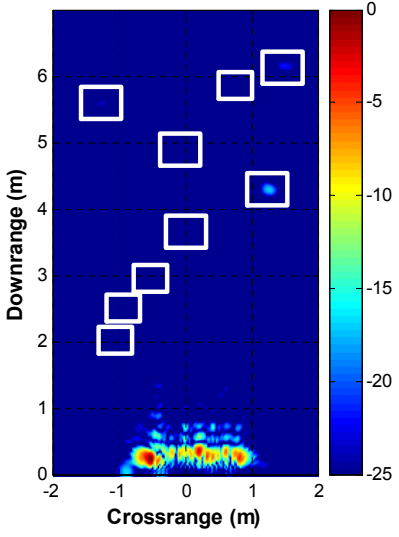

(b)

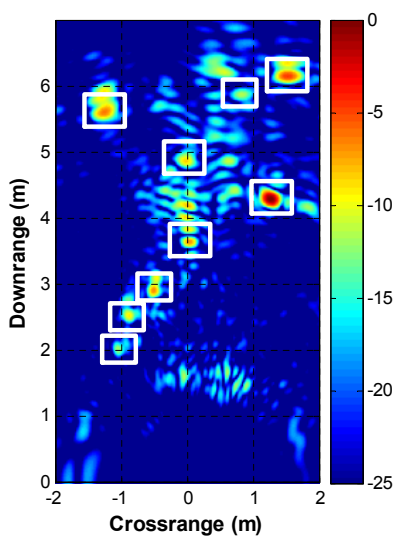

(d)

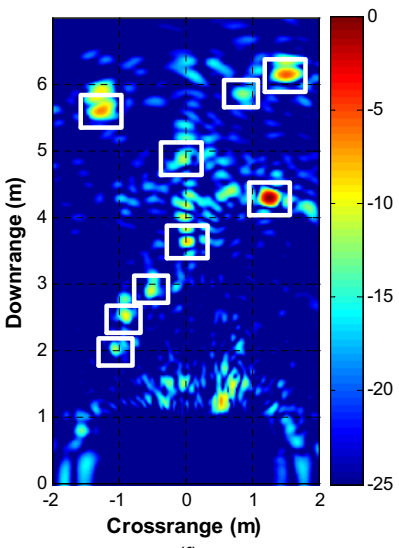

(f)
Fig. 3. Images of the drywall scene obtained using different wall clutter mitigation methods: (a) background subtraction, (b) spatial filtering, (c) entropy-based method, (d) DPSS-based method, (e) SVD-based method and (f) proposed tensor-based method.

\section{REFERENCES}

[1] F. Ahmad, M. G. Amin, and S. A. Kassam, "Synthetic aperture beamformer for imaging through a dielectric wall," IEEE Transactions on Aerospace and Electronic Systems, vol. 41, no. 1, pp. 271-283, 2005.

[2] F. Soldovieri and R. Solimene, "Through-wall imaging via 
a linear inverse scattering algorithm," IEEE Geoscience and Remote Sensing Letters, vol. 4, no. 4, pp. 513-517, 2007.

[3] Q. Huang, L. Qu, B. Wu, and G. Fang, "UWB through-wall imaging based on compressive sensing," IEEE Trans. Geoscience and Remote Sensing, vol. 48, no. 3, pp. 1408-1415, 2010.

[4] M. G. Amin ( Ed.), Through-the-wall radar imaging, Boca Raton, FL: CRC Press, Dec. 2010.

[5] C. Debes, A. M. Zoubir, and M. G. Amin, "Enhanced detection using target polarization signatures in through-the-wall radar imaging," IEEE Transactions on Geoscience and Remote Sensing, vol. 50, no. 5, pp. 1968-1979, 2012.

[6] V. H. Tang, A. Bouzerdoum, and S. L. Phung, "Two-stage through-the-wall radar image formation using compressive sensing," Journal of Electronic Imaging, vol. 22, no. 2, pp. 021006-1- 021006-10, 2013.

[7] C. H. Seng, A. Bouzerdoum, M. G. Amin, and S. L. Phung, "Probabilistic fuzzy image fusion approach for radar through wall sensing," IEEE Transactions on Image Processing, vol. 22, no. 12, pp. 4938-4951, 2013.

[8] P. Setlur, M. G. Amin, and F. Ahmad, "Analysis of microdoppler signals using linear FM basis decomposition," in Proc. SPIE Radar Sensor Technology X, vol. 6210, pp. 62100M-1 62100M-11, Apr. 17-21, 2006.

[9] F. Ahmad, M. G. Amin, and P. Setlur, "Through-the-wall target localization using dual-frequency CW radars," in Proc. SPIE Sensors, and Command, Control, Communications, and Intelligence (C3I) Technologies for Homeland Security and Homeland Defense V, vol. 6201, pp. $62010 \mathrm{H}-1-62010 \mathrm{H}-12$, Apr. 17-21 2006.

[10] Y. Kim and H. Ling, "Human activity classification based on micro-doppler signatures using a support vector machine," IEEE Transactions on Geoscience Remote Sensing, vol. 47, no. 5, pp. 1328-1337, 2009.

[11] M. Dehmollaian and K. Sarabandi, "Refocusing through building walls using synthetic aperture radar," IEEE Transactions on GeoScience and Remote Sensing, vol. 46, no. 6, pp. 15891599, 2008.

[12] C. Thajudeen, A. Hoorfar, and W. Zhang, "Estimation of frequency dependent parameters of unknown walls for enhanced through-the-wall imaging," in Proc. IEEE International Symposium on Antennas and Propagation, pp. 3070-3073, July 38, 2011.

[13] M. Dehmollaian and K. Sarabandi, "Analytical, numerical, and experimental methods for through-the-wall radar imaging," in Proc. IEEE International Conference on Acoustics, Speech and Signal Processing, pp. 5181-5184, Mar. 31-Apr. 4, 2008.

[14] Y.-S. Yoon and M. G. Amin, "Spatial filtering for wall-clutter mitigation in through-the-wall radar imaging," IEEE Transactions on Geoscience and Remote Sensing, vol. 47, no. 9, pp. 3192-3208, 2009.

[15] P. K. Verma, A. N. Gaikwad, D. Singh, and M. J. Nigam, "Analysis of clutter reduction techniques for through wall imaging in UWB range," Progress in Electromagnetics Research B, vol. 17, pp. 29-48, 2009.

[16] F. H. C. Tivive, A. Bouzerdoum, and M. G. Amin, "An SVDbased approach for mitigating wall reflections in through-thewall radar imaging," in Proc. IEEE Radar Conference, pp. 519-524, May 23-27, 2011.
[17] F. H. C. Tivive, M. G. Amin, and A. Bouzerdoum, "Wall clutter mitigation based on eigen-analysis in through-the-wall radar imaging," in Proc. International Conference on Digital Signal Processing, pp. 1-8, July 6-8, 2011.

[18] M. M. Riaz and A. Ghafoor, "Through-wall image enhancement based on singular value decomposition," International Journal of Antennas and Propagation, vol. 2012, pp. 1-20, 2012.

[19] F. H. C. Tivive and A. Bouzerdoum, "An improved SVDbased wall clutter mitigation method for through-the-wall radar imaging," in Proc. IEEE 14th Workshop on Signal Processing Advances in Wireless Communications (SPAWC), pp. 430-434, June 16-19, 2013.

[20] F. H. C. Tivive, A. Bouzerdoum, and M. G. Amin, "A subspace projection approach for wall clutter mitigation in through-thewall radar imaging," IEEE Transactions on Geoscience and Remote Sensing, vol. 53, no. 4, pp. 2108-2122, 2015.

[21] Raffaele Solimene and Antonio Curraro, "Front wall clutter rejection methods in TWI," IEEE Geoscience and Remote Sensing Letters, vol. 11, no. 6, pp. 1158-1162, 2014.

[22] F. Ahmad, J. Qian, and M. G. Amin, "Wall clutter mitigation using discrete prolate spheroidal sequences for sparse reconstruction of indoor stationary scenes," IEEE Transactions on Geoscience and Remote Sensing, vol. 53, no. 3, pp. 15491557, 2014.

[23] NAVY SBIR FY08.1 Solicitation, Radio Frequency (RF) Modeling of Layered Composite Dielectric Building Materials, pp. 94-95. Available online: http://www.acq.osd.mil/osbp/sbir/solicitations/sbir20081/ navy081.pdf

[24] L. D. Lathauwer, B. D. Moor, and J. Vandewalle, "A multilinear singular value decomposition," SIAM. J. Matrix Anal. and Appl., vol. 21, no. 4, pp. 1253-1278, 2000.

[25] M. Haardt, F. Roemer, and G. Del Galdo, "Higher-order SVDbased subspace estimation to improve the parameter estimation accuracy in multidimensional harmonic retrieval problems," IEEE Transactions on Signal Processing, vol. 56, no. 7, pp. 3198-3213, 2008.

[26] A. Rajwade, A. Rangarajan, and A. Banerjee, "Image denoising using the higher order singular value decomposition," IEEE Transactions on Pattern Analysis and Machine Intelligence, vol. 35, no. 4, pp. 849-862, 2012.

[27] N. Liu, B. Zhang, J. Yan, Z. Chen, W. Liu, F. Bai, and L. Chien, "Text representation: from vector to tensor," Fifth IEEE International Conference on Data Mining, pp. 725-728, nov. 2730, 2005.

[28] T. Kolda and B. Bader., "The TOPHITS model for higherorder web link analysis," Proc. SIAM Data Mining Conference Workshop on Link Analysis, Counterterrorism and Security, pp. 725-728, nov. 27-30, 2006.

[29] F. Ahmad, Y. Zhang, and M. G. Amin, "Three-dimensional wideband beamforming for imaging through a single wall," IEEE Geoscience and Remote Sensing Letters, vol. 5, no. 2, pp. 176-179, 2008.

[30] R. Dilsavor, W. Ailes, P. Rush, F. Ahmad, W. Keichel, G. Titi, and M. Amin, "Experiments on wideband through the wall imaging," in Proc. SPIE Symposium on Defense and Security, Algorithms for Synthetic Aperture Radar Imagery XII Conference, vol. 5808, pp. 196-209, Mar. 28-31, 2005. 